\title{
Pericardial Effusion Uncovering Underlying Hypothyroidism
}

\author{
Anoosha Ponnapalli ${ }^{1}$, Smit Deliwala $^{1}$, Yazan Zayed ${ }^{1}$, and Ghassan Bachuwa ${ }^{1}$ \\ ${ }^{1}$ Hurley Medical Center
}

June 8, 2020

\begin{abstract}
Primary hypothyroidism has been associated with many adverse effects. Its cardiovascular manifestations include reductions in heart rate and contractility with the development of hypertension, angina, heart failure, and pericardial effusion consistent with a hypothyroid heart. Early recognition and treatment can help mitigate these advanced features.
\end{abstract}

\section{Key Clinical Message:}

Pericardial effusion may be the initial tipping point to unravel hypothyroidism. Medical therapy over surgical intervention is appropriate if no evidence of cardiac tamponade.

Keywords: Hypothyroidism, pericardial effusion, myxedema, pericardial disease

\section{Case history:}

Question: What are the cardiac implications of untreated hypothyroidism?

Answer: We present a case of pericardial effusion from primary hypothyroidism.

A 70-year-old female with a history of chronic obstructive pulmonary disease (COPD), chronic arthritis with no documented hypothyroidism presented to the emergency department with dyspnea evolving over the last month, generalized weakness, and arthritic pain. Initial vitals, were notable for bradycardia and hypoxia, with an oxygen saturation of $80 \%$ necessitating supplemental oxygen. She was awake, appeared frail, and was not in acute distress. The exam revealed muffled heart sounds, jugular venous distension, and features consistent generalized myxedema (figure 1). Initial workup revealed pancytopenia with macrocytosis and an unremarkable chemistry panel with a brain natriuretic peptide (BNP) of 133.4 PG/ML. Imaging revealed cardiomegaly and pulmonary congestion on chest radiography (figure 2), while computerized tomography (CT) chest with contrast revealed a moderate to large pericardial effusion (figure 3 ). Thyroid-stimulating hormone (TSH) was 102.94 UIU/ML (normal range 0.30-5.50 UIU/ML) with a free $\mathrm{T}_{4}$ less than $0.1 \mathrm{NG} / \mathrm{DL}$ (normal range 0.9-1.8 NG/DL). A transthoracic echocardiogram confirmed a sizable pericardial effusion without tamponade physiology and severe concentric left ventricular hypertrophy (figure 4). She was started on high dose intravenous levothyroxine and hydrocortisone. She was discharged on an oral course of levothyroxine with close follow-up with a cardiologist.

\section{Author Contributions:}

Anoosha Ponnapalli - Design, data collection, and review

Smit S. Deliwala - Data collection, manuscript preparation, and review

Yazan Zayed - Review

Ghassan Bachuwa - Review

Conflict of Interest: None to declare 
Funding: None to declare

\section{References:}

Chahine J, Ala CK, Gentry JL, Pantalone KM, Klein AL. Pericardial diseases in patients with hypothyroidism. Heart . 2019;105(13):1027-1033. doi:10.1136/heartjnl-2018-314528

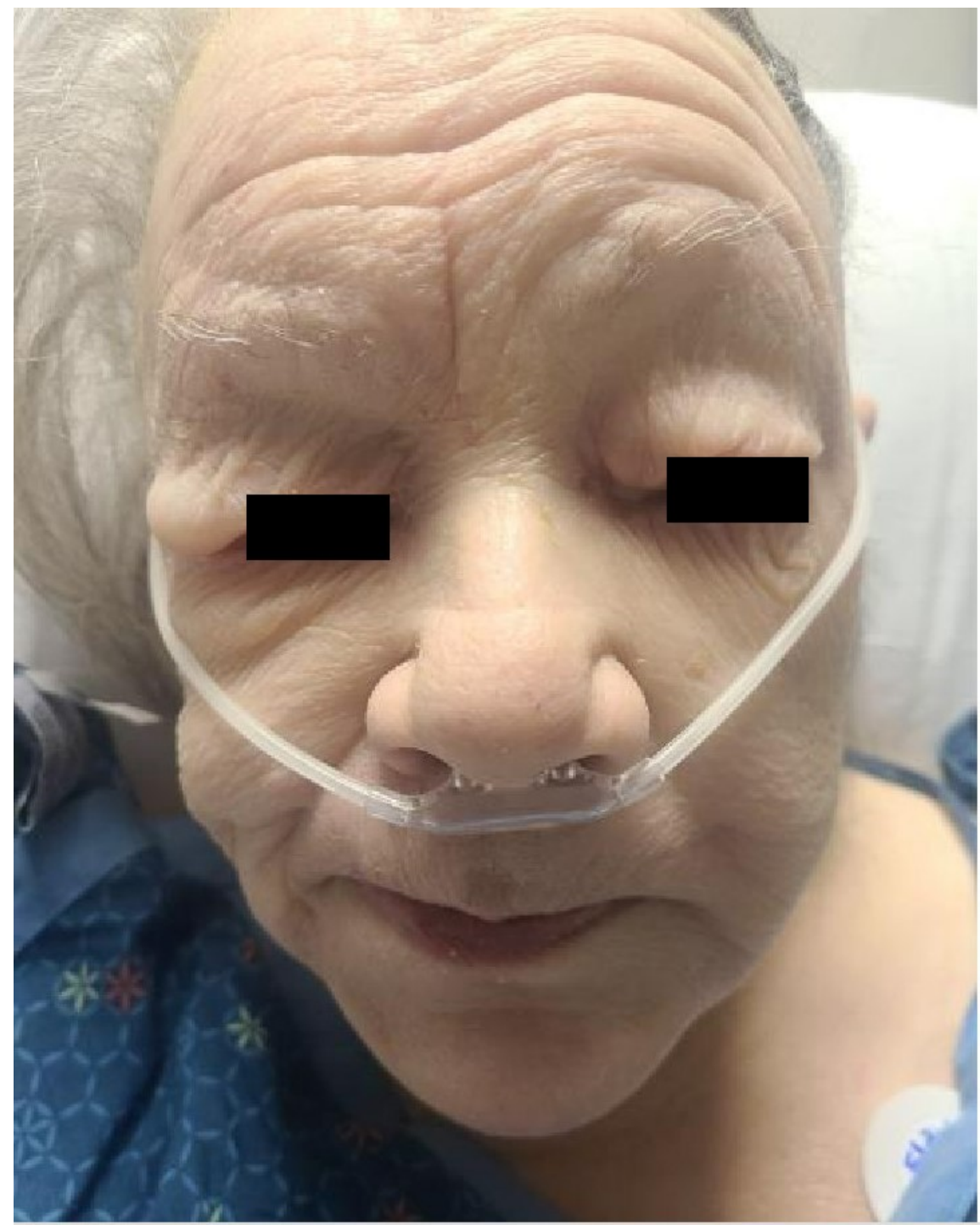




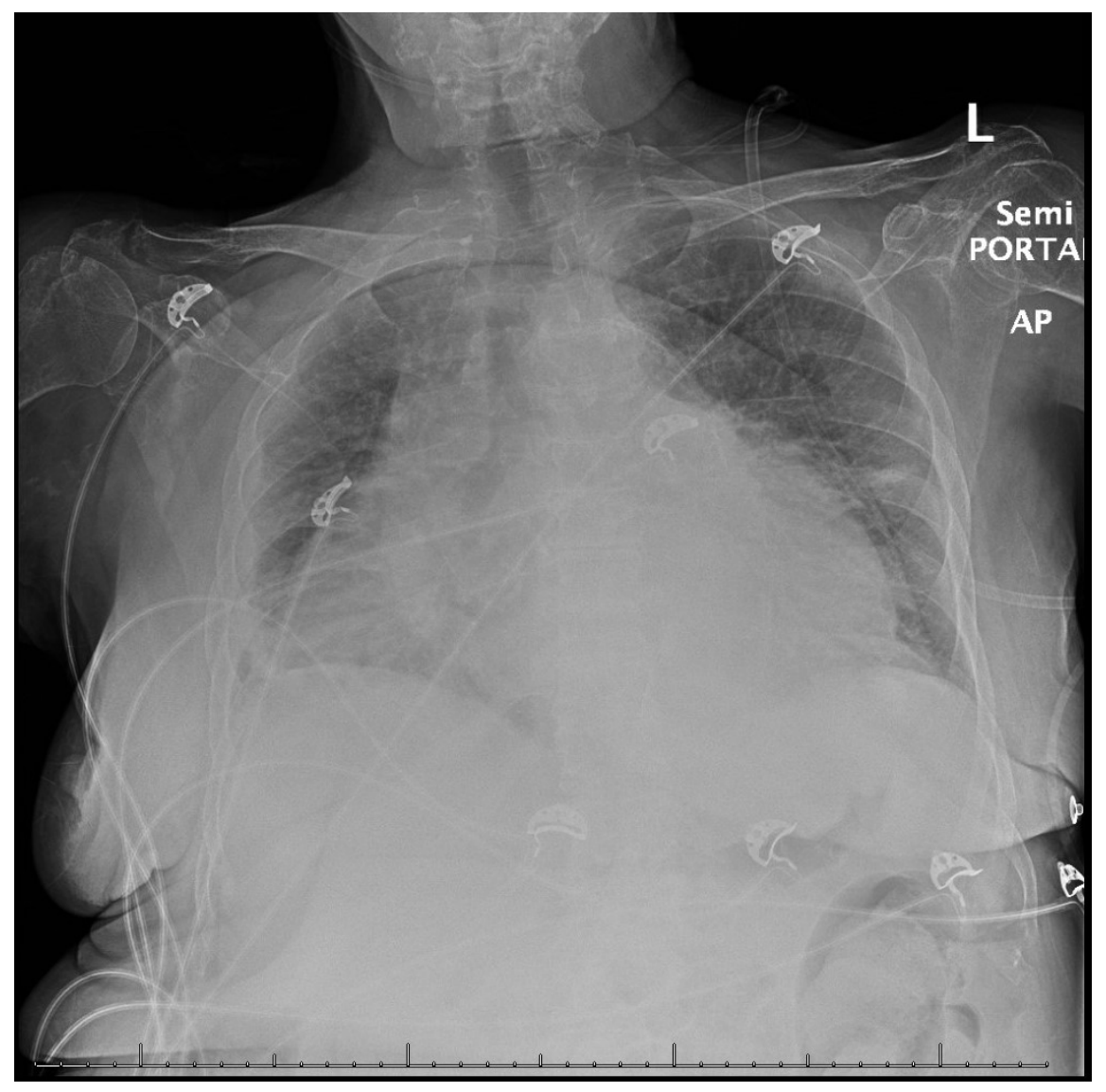



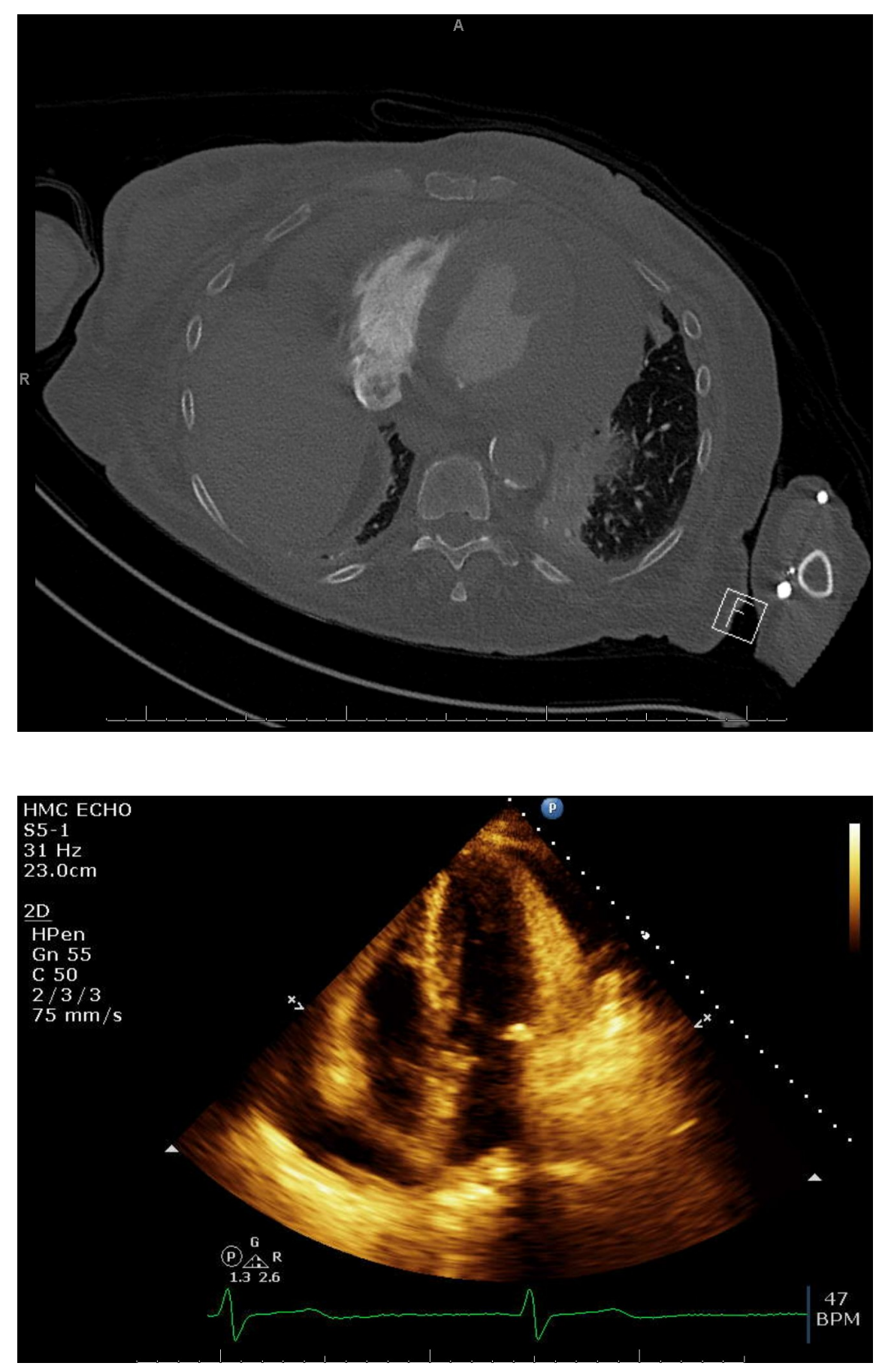